\title{
Stage Unknown
}

National Cancer Institute

\section{Source}

National Cancer Institute. Stage Unknown. NCI Thesaurus. Code C92207.

A finding indicating that the extent of org an/tissue involvement by a malignant neoplasm

is not reported or not assessed. 